\title{
LOCALIZED LANGMUIR EIGENMODES AND SOLAR RADIO BURSTS
}

\author{
D. M. Malaspina*, S. H. Hess*, and R. E. Ergun*†
}

\begin{abstract}
Observed spatial- and frequency-domain signatures of the most intense solar wind Langmuir waves can be described as localized, discrete-frequency eigenmodes trapped in a parabolic density fluctuation. Electric field waveforms from spacecraft in the solar wind are compared with one- and three-dimensional solutions and, in many cases, can be represented by 1-3 of the lowest order eigenmodes. The spatial scale of eigenmode wave packets is on the order of tens of Langmuir wavelengths, allowing them to draw energy directly from the unstable electron distributions associated with a solar type III radio bursts and implying that Langmuir waves can grow in a strongly inhomogeneous medium. The currents generated by localized Langmuir eigenmodes emit coherent electromagnetic radiation as antennas at the fundamental and at twice the local plasma frequency. STEREO observations demonstrate that the currents required for eigenmode antenna radiation are present and have strengths within an order of magnitude of theoretical predictions. The eigenmode antenna radiation mechanism implies that, of all the Langmuir waves excited by an electron beam, relatively few localized antenna radiators may account for a majority of observed emission from an extended radio source. Finally, the possibility that turbulence may ultimately play a strong role in the generation of Langmuir waves and the radio emissions associated with solar type II and type III radio bursts is investigated.
\end{abstract}

\section{Introduction}

Type II and type III solar radio bursts are characterized by a sudden onset of radio emission at 100's of MHz that decreases in frequency over a few hours, eventually reaching the plasma frequency $\left(f_{p} \approx 20 \mathrm{kHz}\right)$ at 1 AU. Type III radio bursts fall smoothly to $f_{p}$, while type II radio bursts have patchy profiles and drop to $f_{p}$ more slowly. Ginsburg and Zhelezniakov [1958] theorized that radiating Langmuir waves excited by beam-unstable electron distributions are responsible for solar radio bursts. Simultaneous observation of Langmuir waves and beam-unstable electron distributions [Lin et al., 1981] in the solar wind

\footnotetext{
* Laboratory for Atmospheric and Space Physics, University of Colorado, Boulder, CO 80309, USA

$\dagger$ Department of Astrophysical and Planetary Sciences, University of Colorado, Boulder, CO 80309, USA
} 
strongly support the primary aspects of this model. Other aspects of the model remain active topics of research, such as determining the mechanism that controls Langmuir wave modulation and amplitude, determining the mechanism that converts electrostatic Langmuir waves into electromagnetic radiation at $f_{p}$ and $2 f_{p}$, and determining the influence of turbulent solar wind plasma on radio burst generation.

To make progress on these topics, the current authors make use of observations from the STEREO mission. The STEREO mission consists of twin spacecraft in heliocentric orbits near 1 AU, one ahead of Earth's orbit (STEREO-A, STA) and one behind Earth's orbit (STEREO-B, STB). Each year the spacecraft gain an angular separation of $\sim 22$ degrees relative to the Sun-Earth line. Each spacecraft has three orthogonal, unbiased $6 \mathrm{~m}$ antennas, described in detail by [Bougeret et al., 2008]. We utilize the Time Domain Sampler (TDS) subsystem, which makes rapid (125k Samples per second $(\mathrm{S} / \mathrm{s}))$, broadband $(\sim 300$ $\mathrm{Hz}-64 \mathrm{kHz})$ voltage measurements. The TDS utilizes on-board event selection logic, telemetering only the highest amplitude events to ground.

In the remainder of this paper, we discuss the modulation of localized Langmuir waves as eigenmodes of solar wind density cavities, the conversion of eigenmode-localized Langmuir waves to electromagnetic radiation at $f_{p}$ and $2 f_{p}$, and the role of turbulence in the relative production of localized and non-localized Langmuir waves with implications for the generation of radio bursts.

\section{Langmuir-eigenmode Modulation}

Though Langmuir waves in solar radio burst source regions have previously been observed by many spacecraft (ISEE 1-3, Helios, Voyager, Galelio, Ulysses, Geotail, WIND, and Cluster), we use STEREO/TDS observations for two reasons. First, STEREO/TDS captured waveform bursts are 130 milliseconds (ms), ten times longer than on previous missions. Second, STEREO/TDS selects for the highest amplitude events, focusing observation on the Langmuir waves that contribute most to solar radio bursts.

Two classifications of Langmuir wave are evident in STEREO/TDS data. Non-localized waves show ms-scale amplitude variation but relatively steady rms value and weak correlation between wave amplitude and simultaneously observed density structure [Malaspina, 2010]. Localized waves show one or more waveform peaks modulated by a single Gaussianlike envelope. Localized waves also show repeatable modulation and the strongest wave amplitudes are often coincident with solar wind density wells [Malaspina, 2010]. Figure 1 shows an example of a non-localized waveform, as well as a localized waveform and its eigenmode fit.

In light of prior observations in Earth's auroral region [McAdams et al., 1999], Ergun et al. [2008] proposed that localized Langmuir waves can be described as eigenmodes of solar wind density cavities. Approximating that any given solar wind density well can be described as parabolic to first order, Ergun et al. [2008] analytically solved the high-frequency Zakharov equation [Zakharov, 1972] (which describes the response of high frequency plasma waves to slower changes in plasma density) in the presence of such a 

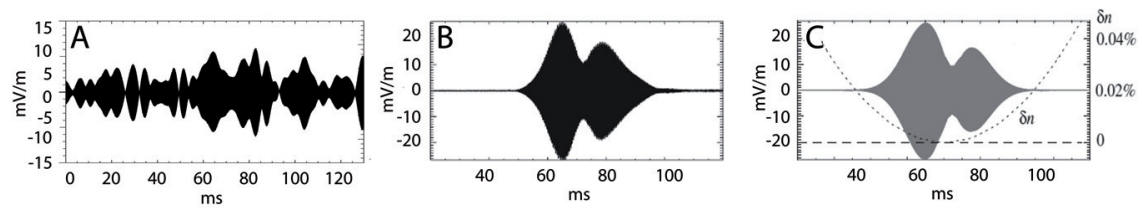

Figure 1: Panel A shows a non-localized Langmuir wave observed by STA on August 18, 2010 at 07:11:26.800. Panel B shows a localized Langmuir wave observed by STB on January 15, 2007 at 17:14:25:248. Panel $C$ shows an eigenmode fit to the wave in panel B, constructed using equation 1. (adapted from Ergun et al. [2008])

well in one dimension. The solutions are Langmuir waves shifted in both frequency and wavenumber $(\Delta \omega, \Delta k)$ modulated by a sum of Hermite-Gauss polynomials $\left(H_{N}(z) e^{-z^{2} / 2}\right)$ as in Equation 1. The Langmuir-eigenmode model accounts for both wave localization and the repeatability of the STEREO/TDS Langmuir wave observations since the waves' spatial extent is limited by the host density cavity size and each localized wave is constructed of the same basis set of electric field modulations with variable relative amplitudes.

$$
E(x, t)=\sum_{N} A_{N} H_{N}(Q x) e^{-Q^{2} x^{2} / 2} e^{i(k+\Delta k) x-i(\omega+\Delta \omega) t}
$$

Here, $\mathrm{x}$ and $\mathrm{t}$ are spatial and temporal coordinates, $A_{N}$ is the fractional strength of each mode, $\mathrm{N}$ is the polynomial order, and $Q^{4}=\omega_{p}^{2} / 6 L^{2} v_{t}^{2}$ for electron plasma frequency $\omega_{p}$, cavity length $L$, and electron thermal velocity $v_{t}$. Detailed expressions for $\Delta k$ and $\Delta \omega$ can be found in Ergun et al. [2008].

Using Equation 1 with the observed solar wind parameters $T_{i}, v_{s w}$, and $\omega_{p}$, and keeping $A_{N}$ and beam speed $v_{b}$ as free parameters, Ergun et al. [2008] reproduced the spatial modulation and frequency-space structure of STEREO/TDS-observed localized Langmuir waves in one dimension using a small number (1-3) of low-order eigenmodes (for one example, see Figure 1C). Malaspina et al. [2008] extended this work to three dimensions, deriving analytic solutions for electric field modulation in both Cartesian and cylindrically symmetric coordinate systems. Though three-dimensional solutions require a larger number of free parameters, the spatial modulations (including the relative phases of perpendicular oscillations) of three-dimensional Langmuir waves were also reproduced.

The Langmuir-eigenmode formalism was extended in Hess et al. [2010a] to include beamdriven growth. It was shown that the modulation and spatial size of an eigenmode wave determine its spread in wavenumber and therefore its ability to extract energy from an electron beam distribution. Lower order modes and spatially larger waves have narrower wavenumber-space profiles and therefore can interact strongly with the narrow positive slope part of the electron beam distribution, resulting in growth to high amplitudes. Higher order modes and spatially smaller waves have broader wavenumber profiles such that only a portion of the component wave numbers draw energy from the driving electron beam distribution while the rest are Landau damped. The conclusion drawn was that lower order modes $(\mathrm{N}=0, \mathrm{~N}=1)$ grow faster and reach higher amplitudes than higher order modes. This result is consistent with localized Langmuir wave observations, which show a 
strong observational predisposition for localized waves with simple modulation structure (few amplitude 'bumps') [Malaspina, 2010].

\section{Langmuir-eigenmode Radiation}

\subsection{Radiation Mechanism}

It is important to determine whether waves modulated according to the Langmuir-eigenmode model described above contribute meaningfully to type II and type III radio emission.

Non-localized Langmuir waves are thought to be converted into electromagnetic radiation through three-wave interactions involving Langmuir, ion-acoustic, and electromagnetic waves (see Robinson and Cairns, [1998] for example). This mechanism requires propagating waves that maintain nearly-constant wave vectors for many Langmuir wavelengths since it relies on momentum-conserving decay to generate $f_{p}$ radiation and the beating of counter-propagating waves to generate $2 f_{p}$ radiation. However, observed Langmuireigenmodes are localized to host density cavities a only few Langmuir wavelengths in extent and so require a different mechanism to radiate efficiently.

We consider instead the antenna radiation of a localized Langmuir-eigenmode. The antenna radiation of localized currents was previously considered by Papadopoulos and Freund [1978] in the context of ponderomotively collapsing Langmuir wave packets, but an observationally consistent form for Langmuir wave modulation was not included. Equation 1 provides the required starting point. To determine the radiation emitted at both $f_{p}$ and $2 f_{p}$ by a Langmuir-eigenmode, two currents are considered, a linear $f_{p}$ current and a weaker nonlinear driven current oscillating at $2 f_{p}$ [Papadopoulos and Freund, 1978]. The general forms for these currents are $j_{f}=i \epsilon_{0} \omega E_{f} \operatorname{Sin}(\omega t)$ and $j_{H}=$ $\left(q_{e} \epsilon_{0} / 2 m_{e} \omega\right) E_{F}\left(\nabla \cdot E_{F}\right) \operatorname{Sin}(2 \omega t)$. From these currents and Equation 1, one can derive near- and far-field electromagnetic fields as well as total power radiated. Full derivations of and expressions for these quantities appear in Malaspina et al. [2010] and Malaspina [2010]. The total radiation generated is found to depend strongly on the peak amplitude of the localized Langmuir wave $\left(\right.$ Power $_{f p} \propto E_{\text {max }}^{2}$ and Power $\left._{2 f p} \propto E_{\text {max }}^{4}\right)$ and the spatial extent of the wave (because antennas are most efficient when the driving current and the emitted radiation wavelength are the same spatial size). Since $j_{H}<<j_{f}$, radiation at $f_{p}$ will dominate in regions where the local Langmuir frequency is high enough to allow $f_{p}$ escape from the eigenmode density cavity (see Malaspina [2010], Chapter 3 for further details).

Observational evidence for localized nonlinear driven currents can be found by examining the spectrograms of individual STEREO/TDS localized Langmuir waveforms (see Figure 2 for an example). Strong wave power is observed at $2 f_{p}$, coincident with peaks in the $f_{p}$ electric field. Malaspina et al., [2010] found order of magnitude agreement between the observed amplitudes of the $2 f_{p}$ electrostatic fields and those predicted using the Papadopoulos and Freund [1978] expression for $j_{H}$. Unlike type III radio bursts, Langmuir-generated radiation from Earth's electron foreshock can be unambiguously identified as $f_{p}$ or $2 f_{p}$. Malaspina et al. [2010] therefore compared WIND observations (tens of Earth radii from 


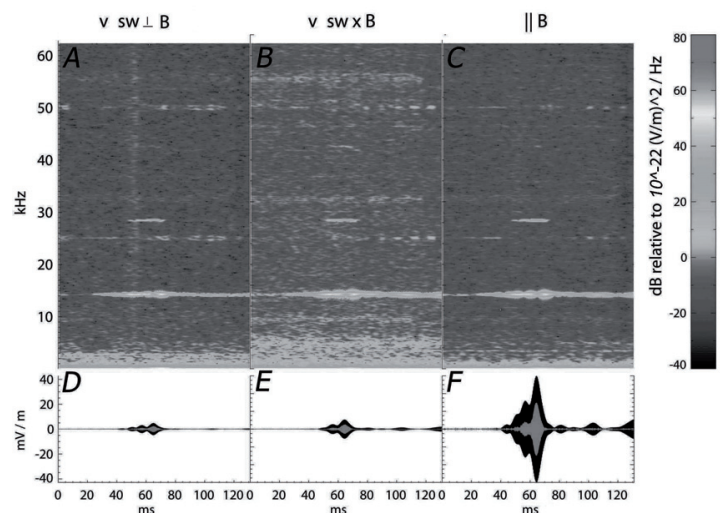

Figure 2: A localized Langmuir wave observed on December 26, 2006 by STA. Panels D, E, and $F$ show the modulation of a single Langmuir wave in three orthogonal directions (directions indicated at the top of the figure). The darker trace shows $f_{p}$ and the lighter, $2 f_{p}$. In these panels, $2 f_{p}$ fields are scaled to half the amplitude of the $f_{p}$ modulations for visibility. Panels $A$, $B$, and $C$ show spectrograms of the waveforms in $D, E$, and $F$. Localized wave power is observed at $f_{p}$ and $2 \mathrm{fp}$. Figure adapted from Malaspina et al. [2010].

the emission region) of $2 f_{p}$ terrestrial foreshock radiation intensity with predictions based on eigenmode antenna emission at $2 f_{p}$. Measured distributions of localized Langmuir wave amplitude and spatial extent were convolved with analytic expressions for radiation intensity and it was found that Langmuir-eigenmode antenna radiation can account for a significant portion of the $2 f_{p}$ radiation from the terrestrial foreshock [Malaspina et al., 2010]. Predictions for the strength of $f_{p}$ radiation are not as straightforward since one must determine the probability that $f_{p}$ radiation can escape from a density well. This complication is treated in Malaspina [2010], section 3.5.

\subsection{Implications for Solar Radio Bursts}

For both localized and non-localized radiation models, electromagnetic power emitted by a Langmuir wave scales as $E^{2}$ for $f_{p}$ radiation and as $E^{4}$ for $2 f_{p}$ radiation, indicating that the highest amplitude waves account for the majority of power radiated by a collection of Langmuir waves.

Using Ulysses data, Nulsen et al. [2007] observed two distinct populations of Langmuir wave while studying the electric field distributions of Langmuir waves in a type III radio burst source region. Below $0.1 \mathrm{mV} / \mathrm{m}$, observed Langmuir wave statistics are consistent with Stochastic Growth Theory (SGT) [Robinson, 1992] which predicts a log-normal distribution of wave amplitudes for non-localized waves in a turbulently fluctuating solar wind. Between $0.1 \mathrm{mV} / \mathrm{m}$ and a few $\mathrm{mV} / \mathrm{m}$, a flatter, non-SGT tail is evident. Nulsen et al. [2007] identify this tail with ILS (Isolated Langmuir Soliton) events. Figure 3 shows an amplitude probability distribution derived by Hess et al. [2010b] from the 
Nulsen et al. [2007] data and STEREO observations of Langmuir-eigenmodes. Scaling this distribution by $E^{2}$ or $E^{4}$ shows that, due to the non-SGT tail at large amplitudes, the highest amplitude events (ILS included) contribute orders of magnitude more radiated power to solar radio bursts then the low amplitude SGT-consistent events. Count rates

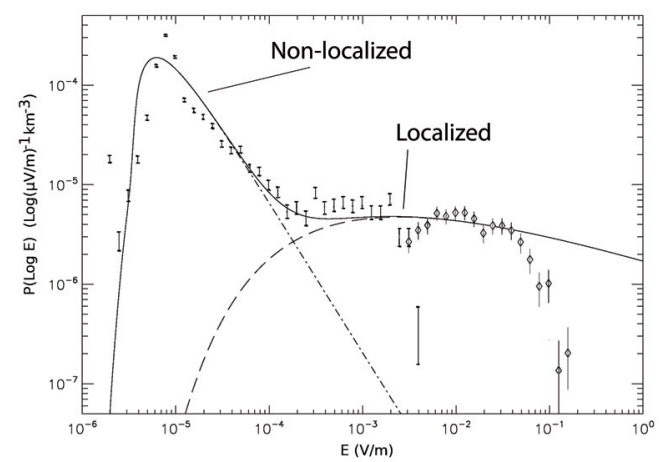

Figure 3: Amplitude probability distribution from Hess et al. [2010b] including the Nulsen et al. [2007] distribution (short dashes with error bars below $2 \times 10^{-3} \mathrm{~V} / \mathrm{m}$ [data used with permission of the authors]) and STEREO observations of localized Langmuir eigenmodes (diamonds with error bars above $2 \times 10^{-3} \mathrm{~V} / \mathrm{m}$ ). Curves of theoretically determined amplitude distributions for non-localized and localized Langmuir waves are plotted over the observed distributions.

between the STEREO and the Nulsen et al. data cannot be compared directly. An arbitrary count rate scaling was introduced for the STEREO data by Hess et al. [2010b] for Figure 3 to illustrate that the shape of the combined distributions is consistent with theoretically derived amplitude probability distributions. Even so, the slope of the nonSGT tail of the Nulsen et al. [2007] distribution is consistent with the slope of the 1-30 $\mathrm{mV} / \mathrm{m}$ portion of the STEREO observations. In this way, both Nulsen et al. and STEREO results indicate the existence of a non-SGT population of Langmuir waves at high amplitudes. Since emitted radiation scales as $E^{2}$ at $f_{p}$ and $E^{4}$ at $2 f_{p}$ for both nonlocalized and localized radiation processes, the highest amplitude waves generate most radio emission from a collection of beam-driven Langmuir waves.

\section{Interactions with Density Turbulence}

For both non-localized and localized Langmuir waves, the wave amplitude distribution is determined by interactions between beam-driven Langmuir waves and the ambient solar wind density turbulence. Recent work by Hess et al. [2010b] explores the growth of both types of beam-driven Langmuir waves in a turbulently fluctuating solar wind. Artificial turbulent density profiles are generated using a sum of sine waves with relative amplitudes chosen to satisfy a Kolmoglorov $k^{-5 / 3}$ spectrum. These profiles are scanned for flat spots and density wells. Flat spots favor the growth of propagating Langmuir waves, while density wells favor the growth of Langmuir-eigenmodes. 
As non-localized Langmuir waves propagate into a region of significantly different density from that in which they formed, their wave vectors will no longer be growth-resonant and they will damp. Since initially growth resonant wave vectors fluctuate strongly with solar wind density variations (see Equation 2, for Langmuir wave vector $k$ and solar wind density $n$ ), a turbulent solar wind with few flat spots will limit the amplitude attainable by non-localized Langmuir waves.

$$
\frac{\Delta k}{k}=\frac{v_{b}^{2}}{6 v_{t}^{2}} \frac{\Delta n}{n}
$$

Langmuir-eigenmode growth is favored wherever the turbulent density profile results in a smooth well (see Hess et al. [2010b] for a detailed description of smoothness criteria). The geometry of the well critically determines which eigenmodes are accessible and what maximum electric field amplitudes may be reached [Hess et al., 2010a].

Starting from a turbulently fluctuating density medium and a theoretical beam-unstable electron distribution, Hess et al. [2010b] determined probability distributions of growth region lengths for localized and non-localized Langmuir waves both numerically and analytically. Using these length distributions, amplitude distributions were then derived based on analytic growth rates. Combining amplitude distributions derived for nonlocalized and localized Langmuir waves, Hess et al. [2010b] reproduced a theoretical amplitude probability distribution consistent with measurements from Nulsen et al. [2007] and scaled measurements from STEREO (Figure 3).

The analytic calculations of Hess et al. [2010b] assume a one dimensional, time stationary model density profile and that the amplitudes reached by non-local Langmuir waves are limited by those waves' dwell time in relatively flat density regions while the amplitudes of localized Langmuir waves are limited by the coherence time of solar wind turbulent density cavities. Hess et al. [2010b] use a simple model for density cavity coherence, but a more detailed model is required since the coherence time of density cavities is critical to determining the maximum amplitudes attainable for localized Langmuir waves. However, at the present time, the problem of determining in detail the coherence time of solar wind turbulent density structures remains unsolved.

\section{Conclusions}

The spatial and frequency domain structure of intense localized solar wind Langmuir waves can be described as discrete-frequency eigenmodes trapped in parabolic density fluctuations. The localization of Langmuir wave currents allows efficient, coherent electromagnetic emission at the local plasma frequency and its first harmonic. Observed Langmuir wave amplitude distributions indicate that many localized waves attain orders of magnitude higher amplitudes than non-localized waves, implying that a relatively small number of Langmuir-eigenmodes radiating as antennas can generate most of the observed radio emission from solar radio bursts. An analytic study of a model turbulent plasma revealed that the magnitude and power spectrum of turbulence in the solar wind determines the available flat spots and density wells, which in turn determine the amplitude 
distribution of local and non-local Langmuir waves, setting the level of total radio burst emission.

\section{References}

Bougeret, J.-L., et al. (42 co-authors), S/WAVES: The Radio and Plasma Wave Investigation on the STEREO Mission, Space Sci. Rev., 136, 487, 2008.

Ergun, R. E., D. M. Malaspina, I. H. Cairns, M. V. Goldman, D. L. Newman, P. A. Robinson, S. Eriksson, J. L. Bougeret, C. Briand, S. D. Bale, C. A. Cattell, P. J. Kellogg, and M. L. Kaiser, Eigenmode Structure in Solar-Wind Langmuir Waves, Phys. Rev. Lett., 101, 051101, 2008.

Ginzburg, V.L., and V.V. Zhelezniakov, To possible mechanisms of sporadic Solar radioemission, Sov. Astron. Zhurn., 35, 694, 1958.

Hess, S. H., D. M. Malaspina, and R. E. Ergun, Growth of the Langmuir cavity eigenmodes in the solar wind, J. Geophys. Res., 115, A10, A10103, 2010a.

Hess, S. H., D. M. Malaspina, and R. E. Ergun, Size and Amplitude of the Langmuir waves in the solar wind, J. Geophys. Res., Submitted, 2010b.

Lin, R. P., D. W. Potter, D. A. Gurnett, and F. L. Scarf, Energetic electrons and plasma waves associated with a solar type III radio burst, Astrophys. J., 251, 364-373, 1981.

McAdams, K. L., R. E. Ergun, and J. LaBelle, HF chirps: Eigenmode trapping in density depletions, Geophys. Res. Lett., 27, 321, 2000.

Malaspina, D. M., Microphysics of the Solar Wind, Ph.D. thesis, University of Colorado, 2010.

Malaspina, D. M. and R.E. Ergun, Observations of three-dimensional Langmuir wave structure, J. Geophys. Res., 113, 12108, 2008.

Malaspina, D. M., I. H. Cairns, and R.E. Ergun, The $2 f_{p}$ radiation from localized Langmuir waves, J. Geophys. Res., 115, 1101, 2010.

Nulsen, A. L., I. H. Cairns, and P. A. Robinson, Field distributions and shapes of Langmuir wave packets observed by Ulysses in an interplanetary type III burst source region, J. Geophys. Res., 112, 5107, 2007.

Papadopoulos, K., and H. P. Freund, Solitons and second harmonic radiation in type III bursts, Geophys. Res. Lett., 5, 881, 1978.

Robinson, P. A., Clumpy Langmuir waves in type III radio sources, Solar Phys., 139, 147, 1992.

Robinson, P. A., and I. H. Cairns, Fundamental and Harmonic Emission in Type III Solar Radio Bursts - I. Emission at a Single Location or Frequency, Solar Phys., 181, 363, 1998.

Zakharov, V.E., Collapse of Langmuir Waves, Sov. J. Exp. Theor. Phys., 35, 908, 1972. 